\title{
CMPK2 accelerates liver ischemia/reperfusion injury via the NLRP3 signaling pathway
}

\author{
YUNHAI LUO, DAOFENG ZHENG, TONG MOU, JUNLIANG PU, \\ ZUOTIAN HUANG, WEI CHEN, YUKE ZHANG and ZHONGJUN WU \\ Department of Hepatobiliary Surgery, The First Affiliated Hospital of Chongqing \\ Medical University, Chongqing 400016, P.R. China
}

Received October 14, 2019; Accepted March 15, 2021

DOI: $10.3892 /$ etm.2021.10793

\begin{abstract}
Cytidine monophosphate kinase 2 (CMPK2) is a mitochondrial nucleotide monophosphate kinase which is important for the substrates of mitochondrial DNA synthesis and has been reported to participate in macrophage activation and the inflammatory response. The purpose of the present research was to determine the potential role of CMPK2 in hepatic ischemia/reperfusion (I/R) injury and to elucidate the underlying molecular mechanisms. The present study investigated the role of CMPK2 in regulating the NLRP3 pathway and liver dysfunction induced by hepatic I/R both in vivo and in vitro. It was revealed that hypoxia/reoxygenation $(\mathrm{H} / \mathrm{R})$ treatment enhanced the mRNA expression levels of CMPK2, NLRP3, IL-18, IL-1 $\beta$ and TNF- $\alpha$ in RAW 264.7 cells. The protein expression levels of IL-18, IL-1 $\beta$ and cleaved-caspase-1 were decreased following CMPK2 knockdown. Furthermore, the inhibition of AIM2 downregulated the expression level of IL-1 $\beta$, IL-18 and cleaved-caspase-1 in the CMPK2 knockdown group followed by $\mathrm{H} / \mathrm{R}$ treatment, while the inhibition of NLRP3 did not. CMPK2 deficiency also decreased alanine aminotransferase and aspartate aminotransferase expression in mice serum, as well as the pathological changes in the liver. Similarly, the release of IL-18 and IL-1 $\beta$ in mouse serum was also restrained with the decline of CMPK2. In conclusion,
\end{abstract}

Correspondence to: Professor Zhongjun Wu, Department of Hepatobiliary Surgery, The First Affiliated Hospital of Chongqing Medical University, 1 Medical College Road, Chongqing 400016, P.R. China

E-mail:wzjtcy@126.com

Abbreviations: CMPK2, cytidine monophosphate kinase 2; mtDNA, mitochondrial DNA; I/R, ischemia/reperfusion; H/R, hypoxia/reoxygenation; H/R, hypoxia/reoxygenation; IL, interleukin; TNF- $\alpha$, tumor necrosis factor- $\alpha$; ALT, alanine aminotransferase; AST, aspartate aminotransferase; NLRP3, NLR family pyrin domain containing 3; AIM2, absent in melanoma 2; KCs, Kupffer cells; LPS, lipopolysaccharide

Key words: CMPK2, hepatic I/R injury, NLRP3 inflammasome, inflammation, AIM2 the results of the present study demonstrate that CMPK2 is indispensable for NLRP3 inflammasome activation, making CMPK2 an effective target to relieve the liver from I/R injury. In addition, the function of CMPK 2 is closely associated with NLRP3 inflammasome activation, instead of AIM2.

\section{Introduction}

I/R injury is a pivotal etiological factor for hepatic dysfunction, and graft failure during liver transplantation and resection (1-3). It has previously been reported that the mechanism of hepatic I/R injury includes necrocytosis, metabolic disturbances and the immune response of inflammation; however, the complete molecular pathology of hepatic I/R injury is yet to be elucidated (4). Kupffer cells (KCs) are identified as liver-settled macrophages and are responsible for identifying and removing exogenous particles and immunoreactive materials that are considered harmful to the human body (5). When activated by liver I/R treatment, KCs adjust the outcome of the immune response in the liver via the regulation of inflammatory signaling pathways $(4,5)$.

The NLRP3 inflammasome, which consists of NLRP3 (a transducer), ASC (an adapter) and caspase-1 (an effector), has been identified as a multiprotein complex $(6,7)$. The stimulation of the NLRP3 inflammasome relies on a special ignition system requiring two necessary processes: Priming and activation. The priming step involves stimulating the synthesis of both NLRP3 and pro-interleukin (IL)-1 $\beta$, which results in a decrease in the threshold of NLRP3 inflammasome activation. The second step, activation, is a poorly characterized event, during which the NLRP3 inflammasome is packaged so as to cleave pro-caspase-1 to mature caspase-1. Once triggered, pro-IL-18 and pro-IL-1 $\beta$ are cleaved by mature caspase-1 to produce functional inflammatory cytokines, namely IL-18 and IL-1 $\beta(6,8)$. Uncontrollable NLRP3 inflammasome is associated with several diseases, such as atherosclerosis (9), sepsis (10), Crohn's disease (11), acute myocardial infarction (12) and cancer (13). Furthermore, recent research has revealed that the NLRP3 pathway is contained in the pathological development of hepatic I/R injury (14-16).

CMPK2, a pyrimidine nucleoside monophosphate kinase, was reported to be in connection with the generation of mitochondrial DNA (mtDNA) (17). CMPK2 is stimulated 
by poly(I:C) and LPS, indicating that CMPK2 has a potent antiviral and antibacterial influence (18-20). Certain other studies have reported the association between CMPK2 and several diseases, such as multiple sclerosis (21), human immunodeficiency virus (22) and Porcine Reproductive and Respiratory Syndrome Virus (23). In addition, CMPK2 has been reported to be involved in the terminal differentiation of monocytes/macrophages (24), macrophage activation and the inflammatory response (17). The mechanism by which CMPK2 plays a part in hepatic I/R injury is yet to be fully elucidated. Therefore, the aim of the present study was to investigate whether CMPK2 affects hepatic I/R injury and to determine the underlying mechanism.

\section{Materials and methods}

Animals. Healthy C57BL/6J male mice ( $\mathrm{n}=43$; weight, 25-28 g; age, 6-8 weeks) were purchased from Chongqing Medical University Laboratory Animals Center. All animal experiments were approved by the Animal Care and Use Committee of Chongqing Medical University. Animal health and behavior was monitored twice a day before operation and once hourly after the operation. All mice used in this study were cared for in strict accordance with the Guide for the Care and Use of Laboratory Animals (25). All mice were raised in a $12 / 12$-h light/dark cycle with $23 \pm 3^{\circ} \mathrm{C}$ and $60-65 \%$ relative humidity and were given a normal diet with free access to food and water.

Hepatic I/R model. A liver I/R model with $70 \%$ ischemia was constructed as previously described (26). Ischemia and reperfusion were performed for 1 and $6 \mathrm{~h}$, respectively. Pentobarbital sodium (intraperitoneal injection, $45 \mathrm{mg} / \mathrm{kg}$ ) was used to anesthetize mice before the laparotomy was performed. The artery and portal vein were occluded to produce the $70 \%$ hepatic ischemia for $1 \mathrm{~h}$. Subsequently, the clamps were removed to perform reperfusion for $6 \mathrm{~h}$. Mice in the sham group underwent an identical surgery but without liver vascular clamping. All mice were euthanized after the operation according to the Guide for the Care and Use of Laboratory Animals. The method of euthanasia used was cervical dislocation. In this study, 40 mice were euthanized after the experiment, 2 mice died from pneumothorax and 1 mouse died following anesthesia. According to AVMA Guidelines for the Euthanasia of Animals and the Guide for the Care and Use of Laboratory Animals (25), mice death was confirmed by recognizing the cessation of vital signs in the mice being euthanized.

Cell culture. RAW 264.7 cells (a murine macrophage cell line) were purchased from Cell Bank of the Cell Bank of Type Culture Collection of the Chinese Academy of Sciences. All cells were cultured in DMEM (Gibco; Thermo Fisher Scientific, Inc.) mixed with $10 \%$ fetal bovine serum (Gibco; Thermo Fisher Scientific, Inc.). Cells were cultured in the cell incubator (Thermo Fisher Scientific, Inc.) at $37^{\circ} \mathrm{C}$ with $95 \%$ air and $5 \% \mathrm{CO}_{2}$.

Cell H/R model. A RAW 264.7 cell H/R model was constructed as previously described (27). Hypoxia was induced for $6 \mathrm{~h}$ by culturing cells in a $37^{\circ} \mathrm{C}$ incubator (Thermo Fisher Scientific, Inc.) with $\mathrm{O}_{2}(1 \%), \mathrm{CO}_{2}(5 \%)$ and $\mathrm{N}_{2}(94 \%)$. The cells were then placed in a $37^{\circ} \mathrm{C}$ incubator with $\mathrm{CO}_{2}(5 \%)$ and air $(95 \%)$ for varying reoxygenation times $(1,6$, 12 and $24 \mathrm{~h})$.

Reverse transcription-quantitative PCR (RT-qPCR) assay. Following the RNAiso Plus (cat. no. 9108; Takara Bio, Inc.) protocol, total RNA was harvested from cells. Reverse transcription was performed by PrimeScript ${ }^{\mathrm{TM}}$ RT reagent Kit (Takara Bio, Inc.) according to the manufacturer's instructions. SYBR $^{\mathrm{TM}}$ Green PCR kit (cat. no. 4309155; Thermo Fisher Scientific, Inc.) was used to detect the relative mRNA expression of CMPK2, NLRP3, IL-1 $\beta$, IL-18 and TNF- $\alpha$. Thermocycling conditions were as follows: Initial denaturation at $95^{\circ} \mathrm{C}$ for $10 \mathrm{~min}$; followed by 40 cycles of $95^{\circ} \mathrm{C}$ for $15 \mathrm{sec}$ and $60^{\circ} \mathrm{C}$ for $60 \mathrm{sec}$. Fold changes were calculated using the $2^{-\Delta \Delta \mathrm{Cq}}$ method (28). mRNA expression data were standardized to $\beta$-actin. The primer sequences were as follows: CMPK2 forward, 5'-GGCAATTATCTCGTGGCTTC-3' and reverse, 5'-GTAGCTATGGCGTAGGTGGC-3'; NLRP3 forward, 5'-GAGTTCTTCGCTGCTATGT-3' and reverse, 5'-GAGTTC TTCGCTGCTATGT-3'; IL-1 $\beta$ forward, 5'-AGTTGACGG ACCCCAAAAGAT-3' and reverse, 5'-GTTGATGTGCTG CTGCGAGA-3'; IL-18 forward, 5'-TGGTTCCATGCTTTC TGGACTCCT-3' and reverse, 5'-TTCCTGGGCCAAGAG GAAGTGATT-3'; TNF- $\alpha$ forward, 5'-CAGGCGGTGCCT ATGTCTC-3' and reverse, 5'-CGATCACCCCGAAGTTCA GTAG-3'; and $\beta$-actin forward, 5'-AGAGGGAAATCGTGC GTGAC-3' and reverse, 5'-CAATAGTGATGACCTGGC CGT-3'. All experiments were repeated in triplicate.

Cell transfection. For the H/R + siCMPK2 group, CMPK2 siRNA was transfected into RAW 264.7 cells using Lipofectamine ${ }^{\circledR} 3000$ (Thermo Fisher Scientific, Inc.), and then cells were transferred to complete medium $6 \mathrm{~h}$ after transfection at $37^{\circ} \mathrm{C}, 24 \mathrm{~h}$ before the H/R treatment, according to the manufacturer's protocol. Similarly, for the $\mathrm{H} / \mathrm{R}+$ scramble siRNA, H/R + siNLRP3, H/R + siAIM2, $\mathrm{H} / \mathrm{R}+\mathrm{NLRP} 3$ siRNA + CMPK2 siRNA and H/R + AIM2 siRNA + CMPK2 siRNA groups, scramble siRNA, NLRP3 siRNA, AIM2 siRNA, NLRP3 siRNA + CMPK2 siRNA and AIM2 siRNA + CMPK2 siRNA were transfected into RAW 264.7 cells in the same way, respectively, and then cells were transferred to complete medium $6 \mathrm{~h}$ after transfection at $37^{\circ} \mathrm{C}$, $24 \mathrm{~h}$ before the H/R treatment.

CMPK2, NLRP3, AIM2 and scramble siRNA were obtained from Shanghai GenePharma Co., Ltd. The sequences were as follows: CMPK2 sense, 5'-GGCAAUUAUCUCGUG GCUUTT-3' and antisense, 5'-AAGCCACGAGAUAAUUGC CTT-3'; NLRP3 sense, 5'-CAACAGGAGAGACCUUUAUTT-3' and antisense, 5'-AUAAAGGUCUCUCCUGUUGTT-3'; AIM2 sense, 5'-GCAGUGACAAUGACUUUAATT-3' and antisense, 5'-UUAAAGUCAUUGUCACUGCTT-3'; scramble siRNA sense, 5'-UUCUCCGAACGUGUCACGUTT-3' and antisense, 5'-ACGUGACACGUUCGGAGAATT-3'

siRNA injection into mice. Mice were randomly divided into four groups: Sham group, I/R group, I/R + scramble siRNA group and I/R + CMPK2 siRNA group; with 5 mice in each 
group. With $24 \mathrm{~h}$ before the operation, CMPK2 siRNA and scramble siRNA were injected into mice of the CMPK2 siRNA group and the scramble siRNA group ( $2 \mathrm{mg} / \mathrm{kg}$; dissolved in normal saline; $100 \mu \mathrm{l}$ injection volume), respectively, via the tail vein, and the same volume of saline was injected into mice of the sham group and I/R group via the tail vein.

Determination of hepatic enzyme. Alanine aminotransferase (ALT; cat. no. C009-2-1) and aspartate aminotransferase (AST; cat. no. C010-2-1; both from Nanjing Jiancheng Bioengineering Institute) serum expression levels in mice were measured using special diagnostic kits according to the manufacturer's protocol. A microplate system (BioTek Instruments, Inc.) was applied to measure the sample absorbance at $510 \mathrm{~nm}$.

Hematoxylin \& eosin $(H \& E)$ staining. Hepatic tissues were fixed with paraformaldehyde (4\%) for $24 \mathrm{~h}$ at room temperature and embedded in paraffin. Tissue specimens were then cut into segments (4.5 $\mu \mathrm{m}$ thick). Then, H\&E staining was performed (staining with hematoxylin for $8 \mathrm{~min}$ followed by staining with eosin for $4 \mathrm{~min}$ at room temperature). The tissues were viewed through an optical microscope (Olympus Corporation; magnification, $\mathrm{x} 200)$.

ELISA. Cell supernatant was collected using a sterile tube, centrifuged at $1,000 \times \mathrm{g}$ at $4^{\circ} \mathrm{C}$ for $20 \mathrm{~min}$, and the supernatant was further collected. Blood was collected using a pyrogen- and endotoxin-free test tube. The blood was coagulated naturally at room temperature for $20 \mathrm{~min}$, and then centrifuged at $1,000 \times \mathrm{g}$ at $4^{\circ} \mathrm{C}$ for $20 \mathrm{~min}$ to collect the serum. The levels of IL-18 (cat no. EMC011) and IL-1 $\beta$ (cat no. EMC001b) cytokines in cellular supernatant and mice serum were detected using special kits from Neobioscience Technology Co., Ltd. according to the manufacturer's protocol, and subsequently quantified via a microplate system (BioTek Instruments, Inc.) at $450 \mathrm{~nm}$.

Western blot analysis. Total protein was harvested with RIPA Lysis Buffer (Beyotime Institute of Biotechnology) and diluted with SDS-PAGE Sample Loading Buffer (5X) (Beyotime Institute of Biotechnology). The expression levels of the target proteins were detected using the protein concentration assay kit (Beyotime Institute of Biotechnology). The proteins (50 $\mu \mathrm{g}$ protein/lane) were separated via SDS-PAGE (12\% gel). Subsequently, the target protein was transferred to the PVDF membranes. After $2 \mathrm{~h}$ blocking with skimmed milk (5\%) at $4^{\circ} \mathrm{C}$, the PVDF membranes, which the target proteins had been transferred to, were washed three times with TBST $(0.1 \%$ Tween-20). Subsequently, the membranes were co-incubated overnight at $4^{\circ} \mathrm{C}$ with CMPK2 (cat. no. ab139720; 1:1,000; Abcam), NLRP3 (cat. no. 15101; 1:1,000; Cell Signaling Technology, Inc.), cleaved-caspase-1 (cat. no. sc-398715; 1:1,000; Santa Cruz Biotechnology, Inc.), IL-1 $\beta$ (cat. no. A16288; 1:1,000; ABclonal Biotech Co., Ltd.), IL-18 (cat. no. bs-0529R; 1:1,000; BIOSS), AIM2 (cat. no. sc-515642; 1:1,000, Santa Cruz Biotechnology, Inc.) and $\beta$-actin (cat. no. 66009-1-lg; 1:5,000, ProteinTech Group, Inc.). The PVDF membranes were washed and co-incubated with the matching secondary antibodies (Beyotime Institute of Biotechnology) for $1 \mathrm{~h}$ at $4^{\circ} \mathrm{C}$. BeyoECL Moon (Beyotime Institute of Biotechnology) was used to observe (Bio-Rad Biotechnology) the target protein.

Statistical analysis. All statistical analyses of data were performed using SPSS software (version 20.0; IBM Corp.) and are presented as the mean \pm standard deviation. The experiments in vitro were performed with 3 independent repeats and the experiments in vivo were performed with 5 independent repeats (3 samples in each group). Unpaired Student's t-test was used to calculate the differences between two groups, while one-way ANOVA followed by Bonferroni's post hoc test was used to analyze multiple groups. $\mathrm{P}<0.05$ was considered to indicate a statistically significant difference.

\section{Results}

$H / R$ treatment alters the expression levels of CMPK2 and NLRP3 in cells. In order to determine the impact of CMPK2 on H/R-induced inflammatory response in cells, the present study first detected the CMPK2 levels. CMPK2 (Fig. 1A), NLRP3 (Fig. 1B) and TNF- $\alpha$, IL-1 $\beta$ and IL-18 (Fig. 1C-E, respectively) mRNA expression levels were increased in RAW 264.7 cells compared with the control group. After reoxygenation for $6 \mathrm{~h}$, CMPK2 mRNA levels were significantly higher (Fig. 1A). Therefore, $6 / 6 \mathrm{~h}$ was selected as an appropriate H/R time node (Fig. 1A). The results demonstrated that increased CMPK2 is likely to be associated with liver I/R.

Knockdown of CMPK2 decreases NLRP3-associated inflammation factor. The present study further investigated the molecular mechanisms underlying CMPK2 in $\mathrm{H} / \mathrm{R}$-induced inflammatory model. In order to determine the effect of CMPK2 on H/R-associated inflammation, cells were transfected with CMPK2 siRNA and scramble siRNA. The efficacy of transfection was then evaluated by performing a western blot assay. It was revealed that CMPK2 expression level was decreased following the transfection of CMPK2 siRNA compared with the scramble siRNA (Fig. 2A).

The results of the western blot assay and ELISAs demonstrated that the protein levels of CMPK2, NLRP3, AIM2, cleaved-caspase-1, IL-18, pro-IL-1 $\beta$ and IL-1 $\beta$ in both $\mathrm{H} / \mathrm{R}$ groups and $\mathrm{H} / \mathrm{R}+$ scramble siRNA groups were increased compared with the control group (Fig. 2B-J). Notably, the $\mathrm{H} / \mathrm{R}+\mathrm{CMPK} 2$ siRNA group exhibited decreased expression of CMPK2, cleaved-caspase-1, IL-18 and IL-1 $\beta$ compared with the H/R group; however, there was no change in the protein levels of AIM2, NLRP3 and pro-IL-1 $\beta$, compared with the H/R groups (Fig. 2B-H). The results of the present study indicate that CMPK2 may increase H/R-induced inflammation by activating (but not priming) the NLRP3 inflammasome.

Knockdown of CMPK2 decreases the expression levels of IL-18 and IL-1 $\beta$ by inhibiting the NLRP3 inflammasome, and not by inhibiting AIM2. In order to eliminate the possibility that CMPK2 has an effect on AIM2 expression levels and to investigate the role of CMPK2 in the NLRP3 pathway in 


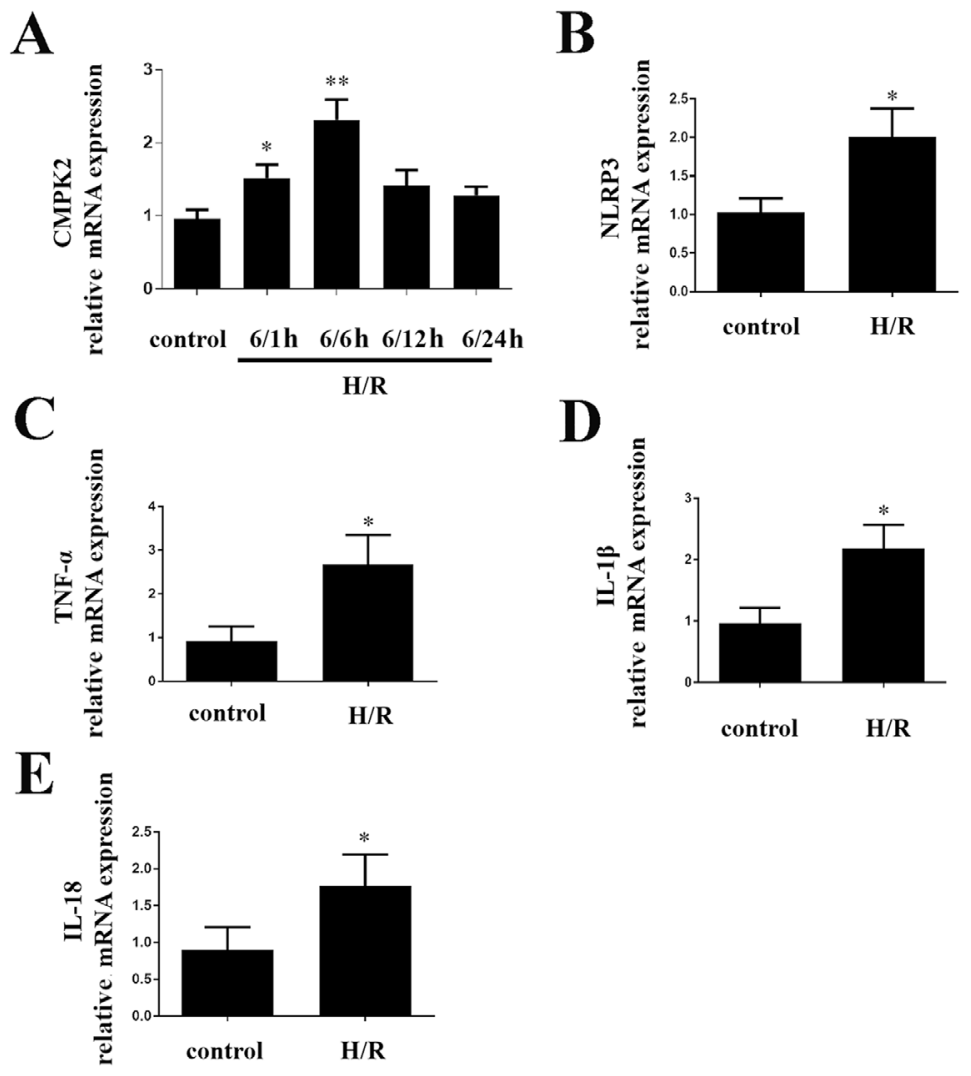

Figure 1. mRNA expression levels of CMPK2, NLRP3 and inflammatory factors in H/R model of RAW 264.7 cells. (A) The mRNA levels of CMPK2 were measured at different time points after reoxygenation. The mRNA expression levels of (B) NLRP3, (C) TNF- $\alpha$, (D) IL-1 $\beta$ and (E) IL-18 were detected following H/R treatment. ${ }^{*} \mathrm{P}<0.05,{ }^{* *} \mathrm{P}<0.01$ vs. control groups. CMPK2, cytidine monophosphate kinase 2 ; NLRP3, NLR family pyrin domain containing 3; H/R, hypoxia/reoxygenation.

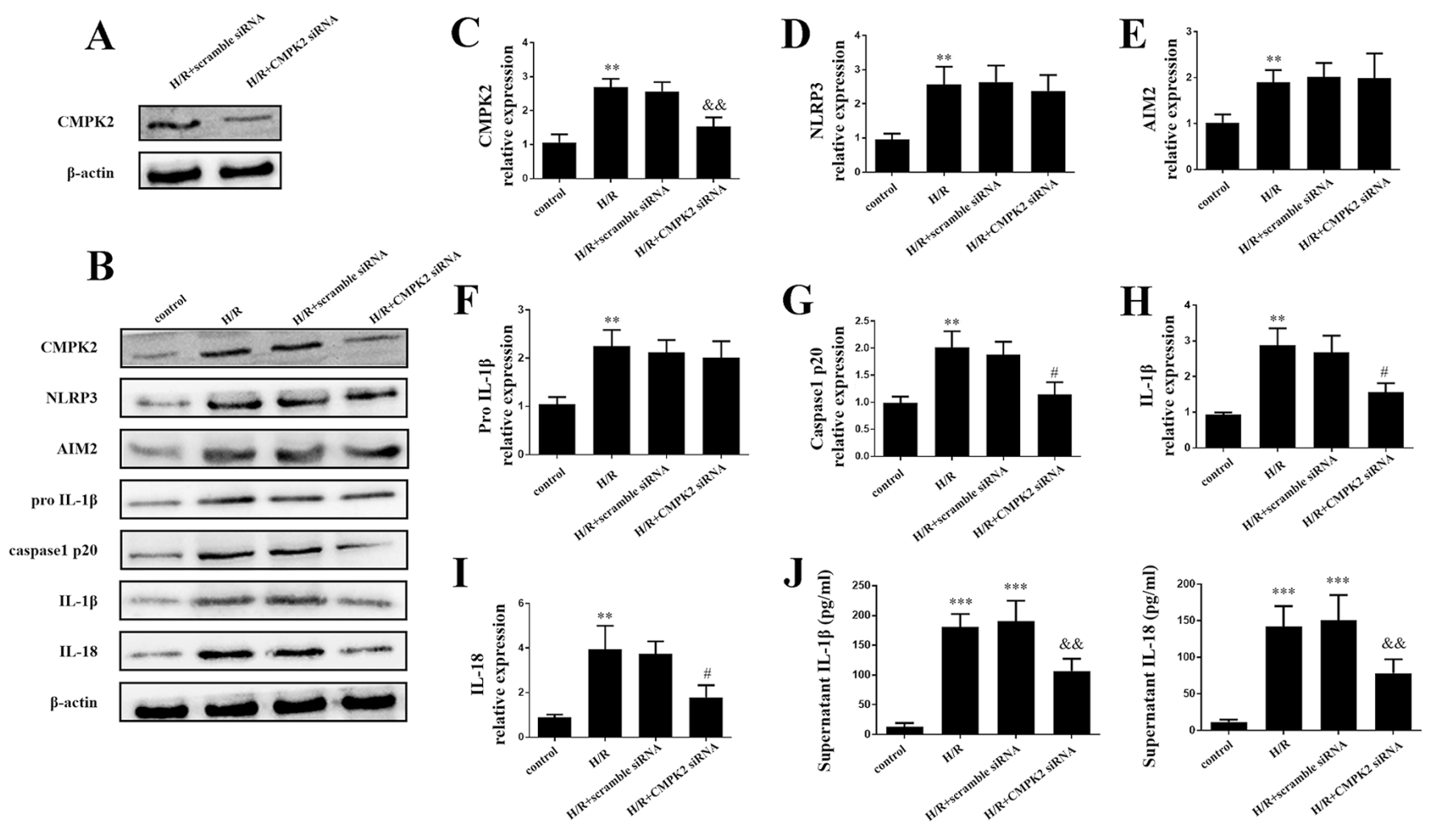

Figure 2. Knockdown of CMPK2 decreased the NLRP3-associated inflammation factor. (A) Protein detection of CMPK2 after H/R treatment in scramble siRNA or CMPK2 siRNA-transfected RAW 264.7 cells. (B) Western blotting and subsequent quantification of (C) CMPK2, (D) NLRP3, (E) AIM2, (F) pro-IL-1 $\beta,(\mathrm{G})$ cleaved-caspase-1 (H) IL-1 $\beta$ and (I) IL-18 protein levels. (J) IL-18 and IL-1 $\beta$ levels in the supernatant were detected by ELISA. ${ }^{* *} \mathrm{P}<0.01$, ${ }^{* * * *} \mathrm{P}<0.001$ vs. control group, ${ }^{*} \mathrm{P}<0.05$, ${ }^{\&}{ }^{\&} \mathrm{P}<0.01$ vs. $\mathrm{H} / \mathrm{R}$ group. CMPK2, cytidine monophosphate kinase 2 ; NLRP3, NLR family pyrin domain containing 3 ; $\mathrm{H} / \mathrm{R}$, hypoxia/reoxygenation; siRNA, small interfering RNA; AIM2, absent in melanoma 2. 
A

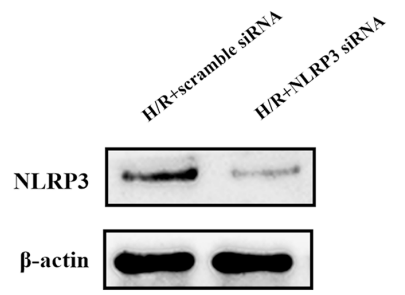

C

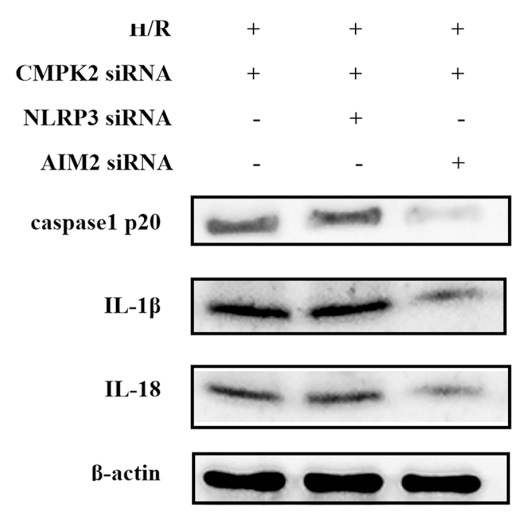

E

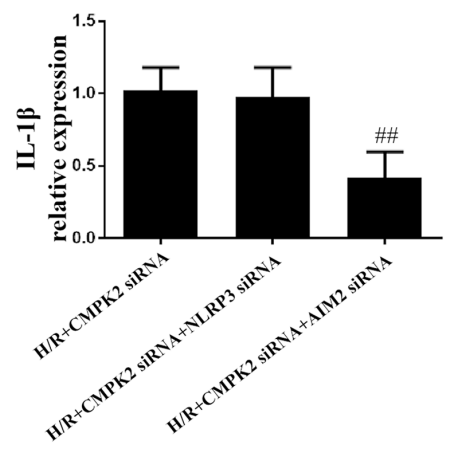

B

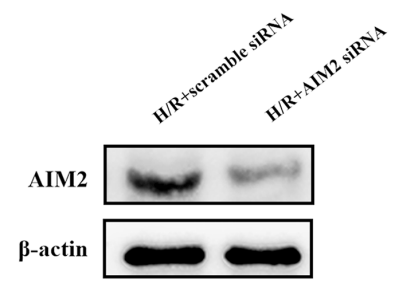

D

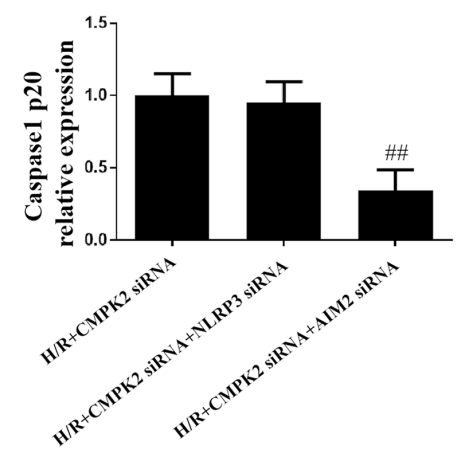

$\mathbf{F}$

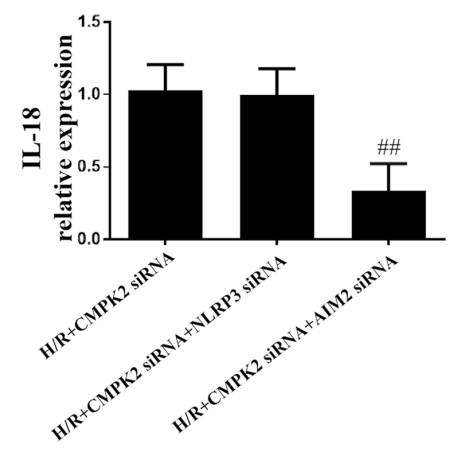

Figure 3. Knockdown of CMPK2 decreases IL-18 and IL-1 $\beta$ by inhibiting the NLRP3 inflammasome, and not AIM2. Protein detection of (A) NLRP3 or (B) AIM2 following H/R treatment in scramble siRNA or specific siRNA-transfected RAW 264.7 cells. (C) Protein detection and respective quantification of (D) cleaved-caspase-1, (E) IL-18 and (F) IL-1 $\beta$ in the H/R + CMPK2 siRNA, H/R + CMPK2 siRNA + NLRP3 siRNA, and H/R + CMPK2 siRNA + AIM2 siRNA groups. ${ }^{\# \#} \mathrm{P}<0.01$ vs. H/R +CMPK2 siRNA group. CMPK2, cytidine monophosphate kinase 2; NLRP3, NLR family pyrin domain containing 3; H/R, hypoxia/reoxygenation; AIM2, absent in melanoma 2; siRNA, small interfering RNA.

H/R-induced inflammation, NLRP3 siRNA and AIM2 siRNA were transfected into cells to knock down the expression of NLRP3 and AIM2, respectively (Fig. 3A and B). As presented in Fig. 3C-F, the protein levels of cleaved-caspase-1, IL-18 and IL-1 $\beta$ in the H/R + CMPK2 siRNA + NLRP3 siRNA groups remained unchanged, compared with the H/R + CMPK2 siRNA groups. Knockdown of CMPK2 + AIM2, but not CMPK2 + NLRP3, suppressed the synthesis of cleaved-caspase-1, IL-18 and IL-1 $\beta$ (Fig. 3C-F). In summary, knockdown of NLRP3, but not AIM2, had no effect on cleaved-caspase-1, IL-18 and IL-1 $\beta$ expression levels following knockdown of CMPK2. This indicates that the knockdown of CMPK2 decreased H/R-induced IL-18 and IL-1 $\beta$ synthesis by targeting the NLRP3 pathway and not AIM2.

Knockdown of CMPK2 alleviates I/R-induced deterioration of hepatic injury. In order to assess the impact of CMPK2 on hepatic I/R induced liver dysfunction, mice were injected with
CMPK2 siRNA or scramble siRNA via the tail vein, followed by hepatic I/R (Fig. 4A). The secretion of IL-18 and IL-1 $\beta$ in mouse serum was measured using an ELISA. It was revealed that treatment with CMPK2 siRNA significantly decreased the secretion of serum IL-18 and IL-1 $\beta$, while treatment with scramble siRNA resulted in an unchanged secretion of serum IL-18 and IL-1 $\beta$, compared with the I/R group (Fig. 4B and $\mathrm{C}$ ). In addition, the present study detected the contents of serum ALT and AST in each group. Compared with the scramble siRNA group, the levels of ALT and AST, which are released following I/R, were significantly inhibited in the CMPK2 siRNA group (Fig. 4D and E). Furthermore, H\&E staining was used to assess the damage following hepatic I/R injury.

Compared with the scramble siRNA group, the amelioration of cellular swelling, bubble degeneration and inflammatory cell infiltration were detected in the CMPK2 knockdown group following hepatic I/R (Fig. 4F and G). 


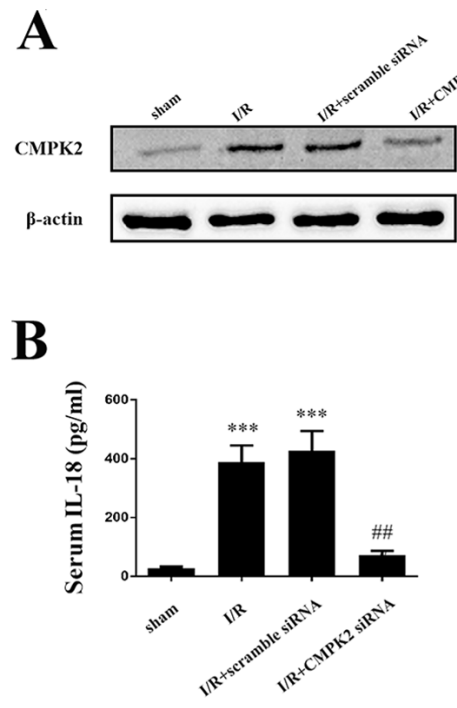

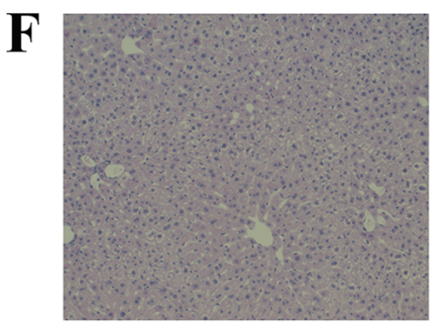

sham

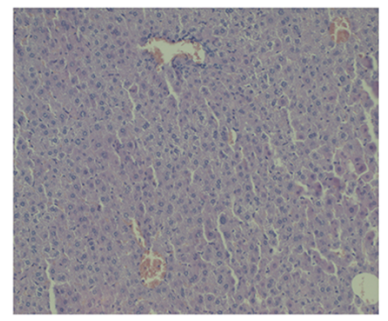

I/R+scramble siRNA

G

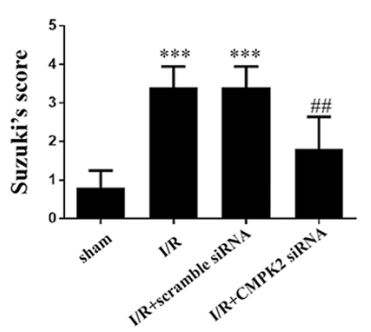

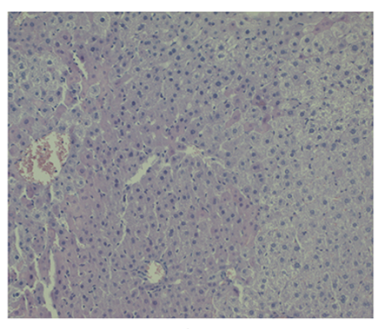

$\mathbf{I} / \mathbf{R}$

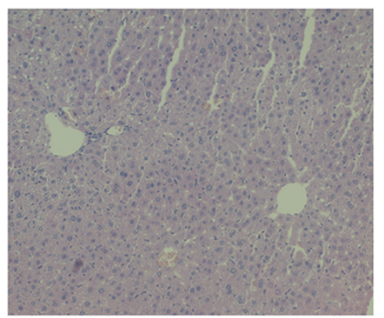

I/R+CMPK2 siRNA
D

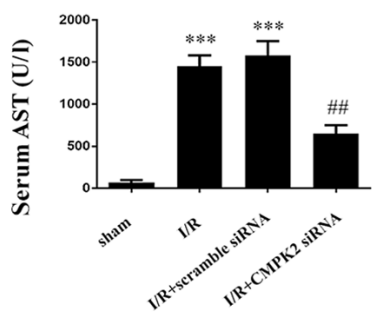

$\mathbf{E}$

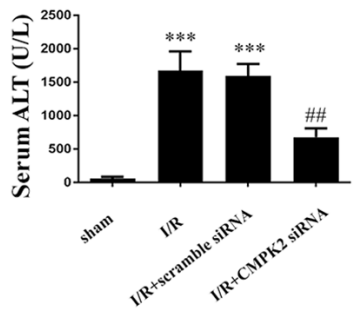

Figure 4. Suppression of CMPK2 alleviates I/R-induced deterioration of hepatic injury. (A) Protein detection of CMPK2 in liver tissue. Secretion of (B) IL-18 and (C) IL-1 $\beta$ was measured by ELISA. Hepatocellular function of experimental mice was evaluated by detecting the release of (D) AST and (E) ALT. ( $F$ and G) Histological changes of the liver in the sham, I/R, I/R + scramble siRNA and I/R + CMPK2 siRNA groups. Original magnification, $x 200 .{ }^{* *} \mathrm{P}<0.01$, ${ }^{* * * * *} \mathrm{P}<0.001$ vs. sham group; ${ }^{\#} \mathrm{P}<0.05,{ }^{\# \#} \mathrm{P}<0.01$ vs. I/R group. CMPK2, cytidine monophosphate kinase 2 ; NLRP3, NLR family pyrin domain containing 3 ; $\mathrm{I} / \mathrm{R}$, ischemia/reperfusion; siRNA, small interfering RNA; ALT, alanine aminotransferase; AST, aspartate aminotransferase.

\section{Discussion}

As a severe complication of liver transplantation, hepatic I/R injury increases the possibility of postoperative infection, transplant failure and mortality rate (29). Previously, several studies have indicated that hepatic I/R injury triggered necrocytosis, metabolic disturbances and the inflammatory immune response $(4,29)$. Among these, the inflammatory immune response has been demonstrated to be a crucial contributor to I/R injury $(4,29)$.

NLRP3 inflammasome has been previously identified as a pivotal regulator in the inflammatory immune response (7). AIM2 inflammasome has also been demonstrated to participate in IL-18 and IL-1 $\beta$ synthesis (30). Although the NLRP3 pathway serves an essential role in several diseases (7), the mechanism that regulates its activation is not yet fully understood. Likewise, the role of the NLRP3 inflammasome in hepatic I/R injury has not yet been fully elucidated. In the present study it was demonstrated that $H / R$ increased the expression level of NLRP3. Furthermore, the activation of NLRP3 inflammasome in both cerebral and cardiac I/R injury has been demonstrated in previous studies $(31,32)$, the results of which are consistent with those of the present study.

In the present study, it was revealed that that $H / R$ treatment enhanced the expression levels of CMPK2, IL-18 and IL-1 $\beta$; the latter two proinflammatory factors were reported to be the final products of the NLRP3 signaling pathway (7). The increased expression of CMPK2 following H/R treatment indicated that CMPK2 may have a potential function associated with the $\mathrm{H} / \mathrm{R}$-induced inflammatory process. In order to confirm the effect of CMPK2 on inflammation, the present study transfected cells with CMPK2 siRNA to decrease the protein levels of CMPK2. Although CMPK2 knockdown did not alter the expression levels of NLRP3 and pro-IL-1 $\beta$, it did decrease the protein levels of cleaved-caspase-1, IL-18 and IL-1 $\beta$ following $H / R$ treatment. In addition, it was revealed that NLRP3 knockdown, but not AIM2 deficiency, had no additional impact on cleaved-caspase-1, IL-18 and IL-1 $\beta$ synthesis in the CMPK2 siRNA group. These results indicated that CMPK2 affected the NLRP3 pathway rather than the AIM2 pathway during hepatic I/R injury, by targeting the NLRP3 pathway. Consistent with the results of the present study, a previous inflammatory model demonstrated that CMPK2 had similar effects on the NLRP3 pathway (8). The present study only focused on the effect of CMPK2 knockdown, and not on CMPK2 overexpression, in hepatic I/R. Future studies will continue to investigate CMPK2 in a comprehensive and in-depth manner.

Previous studies have demonstrated that CMPK2 promoted mtDNA synthesis by providing dCTP (17). Several 
other studies have reported that mtDNA deficiency inhibited cleaved-caspase- 1 and IL-1 $\beta$ production caused by NLRP3 inflammasome activation $(8,33)$. Based on these consequences, it was speculated that the approach of CMPK2-mediated NLRP3 inflammasome activation may be connected with CMPK2-dependent mtDNA synthesis.

To the best of the authors knowledge, the present study reported for the first time that CMPK2 accelerates hepatic I/R injury by activating the NLRP3 inflammasome. CMPK2 siRNA-mediated knockdown not only decreased the NLRP3 inflammasome-mediated inflammatory response, but also relieved hepatic I/R injury. Furthermore, the results of the present study identified a potentially promising therapeutic strategy to alleviate hepatic I/R injury by targeting CMPK2-mediated NLRP3 inflammasome activation.

\section{Acknowledgements}

Not applicable.

\section{Funding}

The present study was funded by the National Natural Science Foundation of China (grant no. 81703063), the National Natural Science Foundation of China (grant no. 81873592) and the graduate tutor team construction project of Chongqing Municipal Education Commission Foundation, China (grant no. dstd201801).

\section{Availability of data and materials}

The datasets used and/or analyzed during the current study are available from the corresponding author on reasonable request.

\section{Authors' contributions}

YL and ZW contributed to the experimental design, data acquisition and preparation of the manuscript. JP, WC and $\mathrm{YZ}$ assessed the liver function and performed the statistical analysis. TM, DZ and ZH contributed to the analysis and interpretation of the data and revised the present article critically for important intellectual content. YL and $\mathrm{ZW}$ confirm the authenticity of all the raw data. All authors read and approved the final manuscript.

\section{Ethics approval and consent to participate}

All experiments were approved by the Animal Care and Use Committee of Chongqing Medical University (Chongqing, China).

\section{Patient consent for publication}

Not applicable.

\section{Competing interests}

The authors declare that they have no competing interests.

\section{References}

1. Peralta C, Jiménez-Castro MB and Gracia-Sancho J: Hepatic ischemia and reperfusion injury: Effects on the liver sinusoidal milieu. J Hepatol 59: 1094-1106, 2013

2. Eltzschig HK and Eckle T: Ischemia and reperfusion-from mechanism to translation. Nat Med 17: 1391-1401, 2011

3. Ni D, Wei H, Chen W, Bao Q, Rosenkrans ZT, Barnhart TE, Ferreira CA, Wang Y, Yao H, Sun T, et al: Ceria nanoparticles meet hepatic ischemia-reperfusion injury: The perfect imperfection. Adv Mater 31: e1902956, 2019.

4. Zhai Y,PetrowskyH,HongJC,BusuttilRWandKupiec-WeglinskiJW: Ischaemia-reperfusion injury in liver transplantation--from bench to bedside. Nat Rev Gastroenterol Hepatol 10: 79-89, 2013.

5. Li P, He K, Li J, Liu Z and Gong J: The role of Kupffer cells in hepatic diseases. Mol Immunol 85: 222-229, 2017.

6. Gross O, Thomas CJ, Guarda G and Tschopp J: The inflammasome: An integrated view. Immunol Rev 243: 136-151, 2011.

7. Swanson KV, Deng M and Ting JP: The NLRP3 inflammasome: Molecular activation and regulation to therapeutics. Nat Rev Immunol 19: 477-489, 2019.

8. Zhong Z, Liang S, Sanchez-Lopez E, He F, Shalapour S, Lin XJ Wong J, Ding S, Seki E, Schnabl B, et al: New mitochondrial DNA synthesis enables NLRP3 inflammasome activation. Nature 560: 198-203, 2018

9. Zhuang T, Liu J, Chen X, Zhang L, Pi J, Sun H, Li L, Bauer R, Wang H, Yu Z, et al: Endothelial Foxp1 suppresses atherosclerosis via modulation of Nlrp3 inflammasome activation. Circ Res 125: 590-605, 2019

10. Martínez-García JJ, Martínez-Banaclocha H, Angosto-Bazarra D, de Torre-Minguela C, Baroja-Mazo A, Alarcón-Vila C, Martínez-Alarcón L, Amores-Iniesta J, Martín-Sánchez F, Ercole GA, et al: P2X7 receptor induces mitochondrial failure in monocytes and compromises NLRP3 inflammasome activation during sepsis. Nat Commun 10: 2711, 2019.

11. Mehto S, Jena KK, Nath P, Chauhan S, Kolapalli SP, Das SK, Sahoo PK, Jain A, Taylor GA and Chauhan S: The crohn's disease risk factor IRGM limits NLRP3 inflammasome activation by impeding its assembly and by mediating its selective autophagy. Mol Cell 73: 429-445.e7, 2019.

12. Mauro AG, Bonaventura A, Mezzaroma E, Quader M and Toldo S: NLRP3 inflammasome in acute myocardial infarction. J Cardiovasc Pharmacol 74: 175-187, 2019.

13. Ghiringhelli F, Apetoh L, Tesniere A, Aymeric L, Ma Y, Ortiz C, Vermaelen K, Panaretakis T, Mignot G, Ullrich E, et al: Activation of the NLRP3 inflammasome in dendritic cells induces IL-1beta-dependent adaptive immunity against tumors. Nat Med 15: 1170-1178, 2009

14. Li C, Jin Y, Wei S, Sun Y, Jiang L, Zhu Q, Farmer DG, Busuttil RW, Kupiec-Weglinski JW and Ke B: Hippo signaling controls NLR family pyrin domain containing 3 activation and governs immunoregulation of mesenchymal stem cells in mouse liver injury. Hepatology 70: 1714-1731, 2019.

15. Miyauchi T,Uchida Y,KadonoK,Hirao H,Kawasoe J, Watanabe T, Ueda S, Okajima $\mathrm{H}$, Terajima $\mathrm{H}$ and Uemoto $\mathrm{S}$ : Up-regulation of FOXO1 and reduced inflammation by $\beta$-hydroxybutyric acid are essential diet restriction benefits against liver injury. Proc Natl Acad Sci USA 116: 13533-13542, 2019.

16. Yue S, Zhu J,Zhang M,Li C,Zhou X,Zhou M, Ke M, Busuttil RW, Ying QL, Kupiec-Weglinski JW, et al: The myeloid heat shock transcription factor $1 / \beta$-catenin axis regulates NLR family, pyrin domain-containing 3 inflammasome activation in mouse liver ischemia/reperfusion injury. Hepatology 64: 1683-1698, 2016.

17. Xu Y, Johansson $M$ and Karlsson A: Human UMP-CMP kinase 2, a novel nucleoside monophosphate kinase localized in mitochondria. J Biol Chem 283: 1563-1571, 2008.

18. Zhang F, Qi Y, Harrison TJ, Luo B, Zhou Y, Li X, Song A, Huang $W$ and Wang Y: Hepatitis E genotype 4 virus from feces of monkeys infected experimentally can be cultured in $\mathrm{PLC} / \mathrm{PRF} / 5$ cells and upregulate host interferon-inducible genes. J Med Virol 86: 1736-1744, 2014.

19. Liu W, Chen B, Chen L, Yao J, Liu J, Kuang M, Wang F, Wang Y, Elkady G, Lu Y, et al: Identification of fish CMPK2 as an interferon stimulated gene against SVCV infection. Fish Shellfish Immunol 92:125-132, 2019.

20. Ueta M, Kawai T, Yokoi N, Akira S and Kinoshita S: Contribution of IPS-1 to polyI: C-induced cytokine production in conjunctival epithelial cells. Biochem Biophys Res Commun 404: 419-423, 2011. 
21. Goertsches RH, Hecker M, Koczan D, Serrano-Fernandez P, Moeller S, Thiesen HJ and Zettl UK: Long-term genome-wide blood RNA expression profiles yield novel molecular response candidates for IFN-beta-1b treatment in relapsing remitting MS. Pharmacogenomics 11: 147-61, 2010.

22. El-Diwany R, Soliman M, Sugawara S, Breitwieser F, Skaist A Coggiano C, Sangal N, Chattergoon M, Bailey JR, Siliciano RF, et al: CMPK2 and BCL-G are associated with type 1 interferon-induced HIV restriction in humans. Sci Adv 4: eaat0843, 2018.

23. Kommadath A, Bao H, Choi I, Reecy JM, Koltes JE, Fritz-Waters E, Eisley CJ, Grant JR, Rowland RR, Tuggle CK, et al: Genetic architecture of gene expression underlying variation in host response to porcine reproductive and respiratory syndrome virus infection. Sci Rep 7: 46203, 2017.

24. Chen YL, Lin DW and Chang ZF: Identification of a putative human mitochondrial thymidine monophosphate kinase associated with monocytic/macrophage terminal differentiation. Genes Cells 13: 679-689, 2008.

25. National Research Council Committee for the Update of the Guide for the $\mathrm{C}$ and Use of Laboratory A: The National Academies Collection: Reports funded by National Institutes of Health. In: Guide for the Care and Use of Laboratory Animals. National Academies Press (US) Copyright (C) 2011. National Academy of Sciences, Washington, DC, 2011.

26. He D, Guo Z, Pu JL, Zheng DF, Wei XF, Liu R, Tang CY and Wu ZJ: Resveratrol preconditioning protects hepatocytes against hepatic ischemia reperfusion injury via Toll-like receptor 4/nuclear factor- $\kappa \mathrm{B}$ signaling pathway in vitro and in vivo. Int Immunopharmacol 35: 201-209, 2016.

27. Wang X, Mao W, Fang C, Tian S, Zhu X, Yang L, Huang Z and $\mathrm{Li} \mathrm{H}$ : Dusp14 protects against hepatic ischaemia-reperfusion injury via Tak1 suppression. J Hepatol S0168-8278: 32275-32284, 2017.
28. Livak KJ and Schmittgen TD: Analysis of relative gene expression data using real-time quantitative PCR and the 2(-Delta Delta C(T)) method. Methods 25: 402-408, 2001.

29. Zhai Y, Busuttil RW and Kupiec-Weglinski JW: Liver ischemia and reperfusion injury: New insights into mechanisms of innate-adaptive immune-mediated tissue inflammation. Am J Transplant 11: 1563-1569, 2011

30. Lugrin J and Martinon F: The AIM2 inflammasome: Sensor of pathogens and cellular perturbations. Immunol Rev 281: 99-114, 2018.

31. He Q, Li Z, Meng C, Wu J, Zhao Y and Zhao J: Parkin-dependent mitophagy is required for the inhibition of ATF4 on NLRP3 inflammasome activation in cerebral ischemia-reperfusion injury in rats. Cells 8: 897, 2019.

32. Darwesh AM, Keshavarz-Bahaghighat H, Jamieson KL and Seubert JM: Genetic deletion or pharmacological inhibition of soluble epoxide hydrolase ameliorates cardiac ischemia/reperfusion injury by attenuating NLRP3 inflammasome activation. Int J Mol Sci 20: 3502, 2019.

33. Zhou R, Yazdi AS, Menu P and Tschopp J: A role for mitochondria in NLRP3 inflammasome activation. Nature 469: 221-225, 2011.

This work is licensed under a Creative Commons Attribution-NonCommercial-NoDerivatives 4.0 International (CC BY-NC-ND 4.0) License. 